\title{
Evaluación agronómica de caucho natural asociado con tres coberturas vegetales en la altillanura
}

\author{
Agronomic evaluation of natural rubber associated \\ with three natural coverages in the altillanura
}

\begin{abstract}
Ibonne Aydee García Romero ${ }^{\circledR}$, Pedro Alberto Garzón ${ }^{2}{ }^{\circledR}$, Maria Elizabeth Méndez Tibambre $^{3}{ }^{\odot}$, Carlos Alberto González Peranquive ${ }^{4}$, Carlos Fernando Cardozo García ${ }^{5}$, Andrés Ricardo Peraza Arias ${ }^{6}$ y Fabio Ancizar Aristizábal Gutiérrez ${ }^{\circledR}$
\end{abstract}

García-Romero, I.A., Garzón, P.A., Méndez-Tibambre, M. E., González-Peranquive, C.A., Cardozo-García, C.F., Peraza-Arias, A.R. y Aristizabal-Gutierrez, F.A. (2020). Evaluación agronómica de caucho natural asociado con tres coberturas vegetales en la altillanura. Colombia forestal, 23(1), 94-108.

Recepción: 20 de junio 2019

\section{Resumen}

Se evaluó el efecto de las coberturas Mucuna bracteata, Desmodium sp. y Pueraria phaseoloides en el crecimiento y su influencia en el estado fitosanitario de un cultivo de caucho natural de dos años de edad en la altillanura colombiana. En condiciones de libre exposición se establecieron tratamientos por cobertura con tres repeticiones y tres réplicas con un control. Las variables respuestas fueron perímetro y la altura total del árbol; se realizaron muestreos del follaje de las coberturas para identificar y cuantificar hongos presentes. Adicionalmente, se hicieron inoculaciones de Colletotrichum gloesporioides en las tres coberturas en condiciones semicontroladas. Se encontró que las tres coberturas generan un leve aumento en la materia orgánica del suelo y favorecen el desarrollo de los árboles. Los hongos más frecuentemente aislados correspondieron a Cladosporium sp., E. sorgui y Fusarium $\mathrm{sp}$. Las inoculaciones mostraron que las tres coberturas son susceptibles a C. gloesporiodes.
Aprobación: 16 de diciembre 2019

Palabras clave: antracnosis, Desmodium sp., Hevea brasiliensis, Mucuna bracteata, Pueraria phaseoloides.

\begin{abstract}
The purpose of this research is evaluating the results of the effect of M. bracteata, Desmodium sp y P. phaseoloides cover crops in growth, and their influence in the phytosanitary conditions of a two years old natural rubber crop in the "Altillanura Colombiana". We established treatments by cover crop with three repeats and a control crop. The development of the trees was evaluated through the measurement of perimeter and total height. Additionally, inoculations of $C$. gloesporioides were carried out in the three cover crops under semi-controlled conditions. It was found that the three cover crops generated a slight increase in the organic matter of the soil and have a favorable effect on the development of the trees. Also, that the most frequently isolated fungi corresponded to Cladosporium sp., E. sorgui and Fusarium
\end{abstract}

\footnotetext{
Universidad Nacional de Colombia. Bogotá, Colombia. iagarciar@unal.edu.co. (Autora responsable de la correspondencia). Grupo Empresarial SYS. Bogotá, Colombia. pealgarzon76@gmail.com.

Corporación Centro de Investigación en Caucho Natural-Cenicaucho. Bogotá, Colombia. memendezt24@gmail.com.

Empresa Mavalle S.A.S. Villavicencio, Colombia. carlos.gonzalez@mavalle.com.

Empresa Mavalle S.A.S. Villavicencio, Colombia. carlos.cardozo@mavalle.com. carlos.cardozo@mavalle.com

Corporación Centro de Investigación en Caucho Natural-Cenicaucho. Bogotá, Colombia. arperazaa@unal.edu.co.

Universidad Nacional de Colombia. Bogotá, Colombia. faaristizabalg@unal.edu.co
} 
sp., and that the inoculations showed that the three coverages are susceptible to C. gloesporioides.
Keywords: antrachnose, Desmodium sp., Hevea brasiliensis, Mucuna bracteata, Pueraria phaseoloides.

\section{INTRODUCCIÓN}

En el cultivo del caucho Hevea brasiliensis (Willd. ex A.Juss.) Müll.Arg. (Euphorbiaceae) se han evaluado diversas especies de leguminosas como coberturas vegetales y se han evidenciado beneficios en todo el ciclo de vida del cultivo, dado que presta servicios como: protección del suelo a la erosión, crea condiciones favorables a la microbiota benéfica, promueve la acumulación de materia orgánica, mejora la estructura física del suelo al tiempo que aumenta la aireación, infiltración y retención de humedad desacelerando su degradación. Su uso también permite minimizar las pérdidas de nutrientes por lixiviación, así como el control de malezas especialmente gramíneas que compiten con el cultivo por los nutrientes (Broughton, 1977; Cherr, Scholberg y McSorley, 2006; Bhaskar y Dey, 2010).

Las características que se buscan de una planta que actúa como cobertura para que su incorporación sea efectiva en el cultivo del caucho son: establecimiento práctico y facilidad de manejo, diseminación y ocupación rápida, persistencia a largo plazo y resistencia al tráfico. Adicionalmente, la cobertura debe tener un sistema radicular que permita un buen anclaje al suelo, pero sin interferir con el sistema radicular de las plantas del cultivo principal; debe aportar nutrientes al suelo; persistir con bajos requerimientos de luz o resistencia a la sombra que le permita crecer después del cierre de las copas de los árboles de Hevea durante los más de 30 años de etapa productiva; y que no favorezcan el desarrollo de problemas fitosanitarios (Fageria, Baligar y Bailey, 2005; Vrignon-Brenas et al., 2019).

Dentro de las especies de leguminosas (Fabaceae) más comúnmente asociadas al cultivo del caucho se encuentran Pueraria phaseoloides (Roxb.) Benth., Mucuna bracteata DC. ex Kurz y Mucuna pruriens (L.) DC. (Samarappuli et al., 2003), también se han usado como coberturas diferentes especies de Desmodium (Pérez-Corpoica et al., 2002). En Colombia se ha promovido el uso de estas tres coberturas en cultivos de caucho de Orinoquía, teniendo en cuenta que los suelos presentan bajo contenido de materia orgánica. Sin embargo, la emergencia de patógenos cosmopolitas en los últimos años, como Colletotrichum spp. causante de la antracnosis en folíolos y panel de rayado de los árboles de caucho (Castro, 2011), ha generado la inquietud sobre si las coberturas vegetales más recomendadas para el cultivo pueden ser o no reservorio de patógenos limitantes para Hevea o verse afectadas por Colletotrichum spp. (Lubbe et al., 2004; Sreenivasaprasad y Talhinhas, 2005).

Este estudio tuvo como objetivo evaluar el efecto en el crecimiento y la fitosanidad del caucho natural en condiciones de campo de las coberturas vegetales $M$. bracteata, Desmodium sp y $P$. phaseoloides, así como la susceptibilidad de estas tres coberturas a C. gloesporioides en condiciones semicontroladas.

\section{MATERIALES Y MÉTODOS}

\section{Análisis del sistema cobertura-cultivo a campo abierto}

Establecimiento de la parcela experimental Se definieron tres lotes, cada uno de una hectárea con árboles RRIM 600 sembrados en el año 2011, con una densidad de 555 árboles. ha-1 en la plantación de Mavalle S. A., ubicada en el municipio de Puerto López (4¹2'13.00"N - 72³4'33.17"W). En cada lote se establecieron cuatro tratamientos correspondientes a las coberturas $M$. bracteata, P. phaseoloides "Kudzu", Desmodium sp. y áreas 
sin cobertura como tratamiento control se manejó un total de 12 unidades experimentales de un tamaño de $250 \mathrm{~m}^{2}$. Para el establecimiento de las coberturas se realizó un arado de rastrillo con la finalidad de disminuir las poblaciones de arvenses establecidas en los lotes, ocho días después se establecieron las coberturas; en el caso de kudzu y Desmodium sp se hizo al voleo con una densidad de siembra de $2.7 \mathrm{~kg}$ de semilla.ha ${ }^{-1}$ de caucho y $1.9 \mathrm{~kg}$ de semilla.ha-1 de caucho respectivamente; para M. bracteata se sembraron tres semillas por sitio a una distancia de $2 \mathrm{~m}$ entre sitio para aproximadamente $450 \mathrm{~g}$ de semilla.ha-1.

\section{Determinación de los efectos en suelo, microambiente y plantas de caucho}

Para establecer el efecto de las coberturas sobre la materia orgánica $(\mathrm{MO})$ y el $\mathrm{pH}$ del suelo se tomaron muestras de cada área por tratamiento antes y 10 meses después del establecimiento de las coberturas, siguiendo la metodología propuesta por Cenicaucho-Corpoica (2013), las cuales fueron llevadas al Laboratorio Nacional de Suelos del Instituto Geográfico Agustín Codazzi (Igac). La MO fue calculada mediante la relación carbono orgánico determinado mediante el método propuesto por Walkley y Black (1934) y el nitrógeno total por Kjeldahl. Para determinar el efecto de las coberturas sobre el desarrollo de los árboles de $\mathrm{H}$. brasiliensis se tomaron los registros de altura total y perímetro a una altura de $1.2 \mathrm{~m}$ con respecto al suelo, de todos los individuos presentes en cada uno de los lotes (150 árboles por tratamiento). Adicionalmente, se realizó la vigilancia fitosanitaria de acuerdo con la metodología prevista por la plantación dentro de sus labores de rutina, que consiste en la evaluación semanal de plagas y enfermedades del $4 \%$ de la plantación a través del muestreo de tres hojas por cada árbol seleccionado (estadios A hasta B2), las cuales son examinadas con lupa.

Para establecer las diferencias de la humedad relativa (HR) y temperaturas (media, máximas y mínimas) en cada tratamiento durante el periodo de evaluación se pusieron en marcha cuatro data-loggers Extech RHT10 dentro de cada una de las parcelas a una altura de $1.20 \mathrm{~m}$, a partir de los tres meses de establecidas las coberturas, con una frecuencia de medición cada 30 minutos.

\section{Determinación de la presencia de potenciales patógenos en las coberturas}

Se tomaron al azar foliolos de cada una de las tres coberturas establecidas, de manera mensual, se preservaron en bolsas de papel y plásticas de polietileno dentro de neveras de polipropileno a una temperatura $10^{\circ} \mathrm{C}$, para su traslado al laboratorio de microbiología del Instituto de Biotecnología de la Universidad Nacional de Colombia (Ibun). Del material recolectado se tomaron $10 \mathrm{~g}$ de material foliar y se dispusieron en $250 \mathrm{ml}$ de agua estéril en un Erlenmeyer de $1000 \mathrm{ml}$, se dejaron $30 \mathrm{~min}$ con agitación periódica. Se extrajo la solución sin sólidos de la cual se realizaron diluciones seriadas hasta $1 \times 10^{-3}$ (previa normalización de la metodología de recuento) y se sembraron $100 \mu \mathrm{l}$ de las diluciones por triplicado en cajas de Petri con PDA y 50 ppm de cloranfenicol. Se hizo seguimiento desde los tres días de sembradas las diluciones hasta ocho días después, se realizó la identificación de las colonias de hongos presentes por microscopia con respecto al género de acuerdo con claves taxonómicas (Gunnell y Gubler, 1992; Leslie, Summerell y Bullock, 2006; Manamgoda et al., 2012; Peberdy, 2013; Manamgoda et al., 2015). Para definir la especie, así como el género de los hongos que no presentaron estructuras reproductivas se realizó el aislamiento de las colonias y la extracción de ADN (Stirling, 2003). A partir del ADN obtenido se hizo la amplificación por PCR de la región ITS (Internal Transcribed Spacer) completa, utilizando los primers ITS1/ITS4 (White et al., 1990) y las condiciones de PCR propuestas por Pérez-Vera et al. (2005). Los fragmentos amplificados fueron secuenciados (Sanger y Coulson, 1975) y, posteriormente, analizados frente a las bases de datos del National Center for Biotechnology Information (NCBI). 


\section{Pruebas de inoculación en condiciones bajo cubierta}

\section{Aislamiento de C. gloesporioides.}

Para obtener aislamientos de C. gloesporioides se tomaron foliolos en estadio $C$ de clones de caucho RRIM 600 y FX 3864 con síntomas de antracnosis descritos por Furtado (2008). Las muestras fueron llevadas al laboratorio de caracterización molecular del Ibun, se tomaron fragmentos de los foliolos de $5 \mathrm{~mm}^{2}$ y se sometieron a desinfección con etanol $70 \%$ por 5 min, hipoclorito $2.5 \%$ por 5 min y dos lavados con agua destilada estéril durante un minuto. Los ejemplares desinfectados bajo condiciones de asepsia se dispusieron sobre papel absorbente estéril para retirar el agua y se sembraron en cajas de Petri con medio PDA (Papa Dextrosa Agar) y 50 ppm de cloranfenicol; se incubaron a $24^{\circ} \mathrm{C}$ en oscuridad por siete días; posteriormente, se realizaron observaciones al microscopio. Los aislamientos que presentaron conidios característicos de algunas especies de Colletotrichum (Weir, Johnston y Damm, 2012) se resembraron en cajas con medio PDA con el fin de purificarlos. Para definir la especie de los aislamientos obtenidos se realizó la extracción de ADN siguiendo la metodología propuesta por Stirling (2003), luego se hizo la amplificación y secuenciación de la región ITS utilizando los primers ITS1/ITS4 (White et al., 1990). Los aislamientos obtenidos fueron depositados en el banco de genes y cepas del Ibun.

\section{Establecimiento de las coberturas en casa malla}

En casa malla, ubicada en la plantación de caucho natural de Mavalle S. A., se sembraron las tres coberturas en cubetas de $30 \times 40 \times 12 \mathrm{~cm}, 12$ cubetas por cada una de las tres coberturas para un total de 36 cubetas, usando como sustrato mezcla de tierra negra de monte y arena en proporción 70:30. Las cubetas fueron regadas dos veces por semana sin ningún esquema de fertilización. Cada cubeta se consideró como unidad experimental por tratamiento.

\section{Inoculaciones}

Tres meses después de la siembra se inoculó a cada unidad experimental con uno de los tres aislamientos de C. gloesporioides obtenidos desde clones de caucho RRIM 600 y FX 3864, utilizando una concentración de conidias para el aislamiento C-1 de $2.3 \times 10^{4}$, C-2 de $2.7 \times 10^{4}$ y C-3 de $2.1 \times 10^{4}$ conidias/ml. Para la obtención de las conidias se repicaron los aislamientos en PDA con cloranfenicol 50 ppm y 15 días después se extrajeron las conidias colocando $10 \mathrm{ml}$ de agua sobre el cultivo y agitación orbital, este procedimiento se repitió tres veces. Después, mediante recuento en cámara de Neubauer, se estableció la concentración de las suspensiones, Unidades Formadoras de Colonias (UFC). Las diluciones para obtener concentraciones mencionadas se realizaron en agua purificada por sistema Milli-Q®. La suspensión de conidios se aplicó con ayuda de un atomizador; por aislamiento se inocularon cuatro cubetas (replicas).

15 días después de la inoculación se determinó la incidencia del patógeno en los foliolos de cinco plántulas de cada una de las coberturas por cubeta, seleccionadas de manera aleatoria; para ello se evaluó la presencia de manchas necróticas características de antracnosis (Furtado, 2008).

La confirmación de la infección del material seleccionado se efectuó mediante evaluación microscópica de la presencia de conidios y análisis de las regiones ITS de aislamientos provenientes de dos foliolos por cada cobertura con síntomas de antracnosis.

\section{Análisis de datos}

Los datos de análisis del suelo y las variables climáticas, así como los datos de perímetro y altura total, se analizaron con el programa SPSS Statistics 2.2 (IBM Corp, 2013). Para los datos de perímetro y altura total se consideró un diseño completamente al azar teniendo en cuenta como tratamientos las tres coberturas establecidas y el control en los dos tiempos de evaluación. También, se utilizó la prueba de homogeneidad de Levene para comprobar el supuesto de homogeneidad de varianzas con un 
$\mathrm{p}<0.05$. Se realizó la prueba de contrastes de Dunnet para definir diferencias entre tratamientos.

Para estimar la incidencia de antracnosis sobre las tres coberturas en condiciones de casa malla se ejecutó un submuestreo de los datos obtenidos, dejando tres por réplica. Se hizo además un análisis de Anova univariante para comprobar la homogeneidad de los datos con un $\mathrm{p}<0.05$, y prueba Tukey para definir diferencias entre tratamientos.

\section{RESULTADOS}

\section{Establecimiento de parcelas en campo}

Desde su siembra las coberturas presentaron competencia con el desarrollo de arvenses, limitando su establecimiento. M. bracteata superó la competencia logrando un cubrimiento del $100 \%$ entre los surcos de $H$. brasiliensis en ocho meses, siendo el menor tiempo comparado a las otras coberturas; $P$. phaseoloides en un periodo cercano a los nueve meses con la realización de deshierbe; y Desmodium sp. presentó competencia con las arvenses al final del ensayo a los 10 meses, sin embargo, las plántulas de esta cobertura persistieron por debajo de las arvenses.

Durante los meses de evaluación la temperatura diaria presentó una tendencia similar en los tratamientos (figuras 1a a 1c) (Levene $\mathrm{p}=0.979$, $\mathrm{F}=2.271, \mathrm{p}=0.079$ ) señalando poca diferencia en el tiempo evaluado. No obstante, el tratamiento Mucuna presentó diferencias con el control para los valores diarios (Dunnet $\mathrm{p}=0.031$ ).

El comportamiento promedio de la temperatura mensual entre los tratamientos fue similar, sin variaciones que permitan establecer diferencias estadísticamente significativas en comparación al control ( $\mathrm{F}=0.166, \mathrm{p}=0.918$ ).

Pese a no existir diferencias estadísticas se observó que la temperatura promedio mensual en el tiempo de evaluación para el testigo presentó un intervalo entre $28.7-25.35{ }^{\circ} \mathrm{C}$ con mediana de $26.93{ }^{\circ} \mathrm{C}$, aproximadamente $0.32 \pm 0.11^{\circ} \mathrm{C}$ por debajo en comparación con los tratamientos con cobertura, por lo que se resalta un comportamiento posiblemente de microclima. Desmodium sp. $\left(29.05-25.66^{\circ} \mathrm{C}\right.$, mediana $\left.=27.16^{\circ} \mathrm{C}\right), M$. bracteata $\left(25.80-29.24^{\circ} \mathrm{C}\right.$, mediana $\left.=27.38^{\circ} \mathrm{C}\right)$ y $P$. phaseoloides $\left(25.68-29.07^{\circ} \mathrm{C}\right.$, mediana $=27.22^{\circ} \mathrm{C}$ ).

La variable de HR (1b) no presentó diferencias entre los tratamientos (Levene $=0.952, \mathrm{~F}=0.048$, $\mathrm{p}=0.986$ ). Sin embargo, la mediana de los datos obtenidos en los tratamientos con cobertura presentó valores mayores que el tratamiento testigo, señalando el posible efecto de microclima. Desmodium sp. (59.43-86.73\%, mediana=82.87\%), Mucuna (57.50-86.69\%, mediana=83.04\%), P. phaseoloides $(60.13-87.17 \%$, mediana $=84.26 \%)$, testigo (56.52-87.32\% mediana=82.16\%).

\section{Análisis de suelos}

El suelo en el que se establecieron los tratamientos corresponde taxonómicamente a un oxisol con régimen de húmedo udico, con horizonte óxico dentro de los $150 \mathrm{~cm}$ de profundidad y más del $40 \%$ de arcilla en los primeros perfiles. Los análisis físicos iniciales mostraron una clase textura franco-arenosa, con un porcentaje promedio de contenidos de arena de $33.13 \% \pm 1.8,28.6 \% \pm 2$ de limo y arcillas de $38.3 \% \pm 1.8$. El análisis químico inicial mostró un pH entre 4.6 y 5.1, con un contenido de materia orgánica inicial promedio de $1.94 \pm 0.2$, un porcentaje de nitrógeno total del $0.079 \pm 0.009$ con una relación $\mathrm{C} / \mathrm{N}$ de $11.62 \pm 0.013$.

El resultado de los análisis de suelos realizados después de 10 meses de establecidas las coberturas mostró variaciones en el $\mathrm{pH}$ en un intervalo de 5.1 a 5.8 sin diferencias estadísticamente significativas entre los tratamientos ( $p=0.952, F=4.128$ ).

En cuanto al contenido de materia orgánica, el análisis descriptivo de los datos presentó una asimetría negativa, por lo tanto, se transformaron los datos aplicando la función $X^{2}$. Se puede inferir que existió una acumulación en el tiempo en todos los tratamientos, con mejores resultados en las áreas con cobertura que en el testigo siendo 

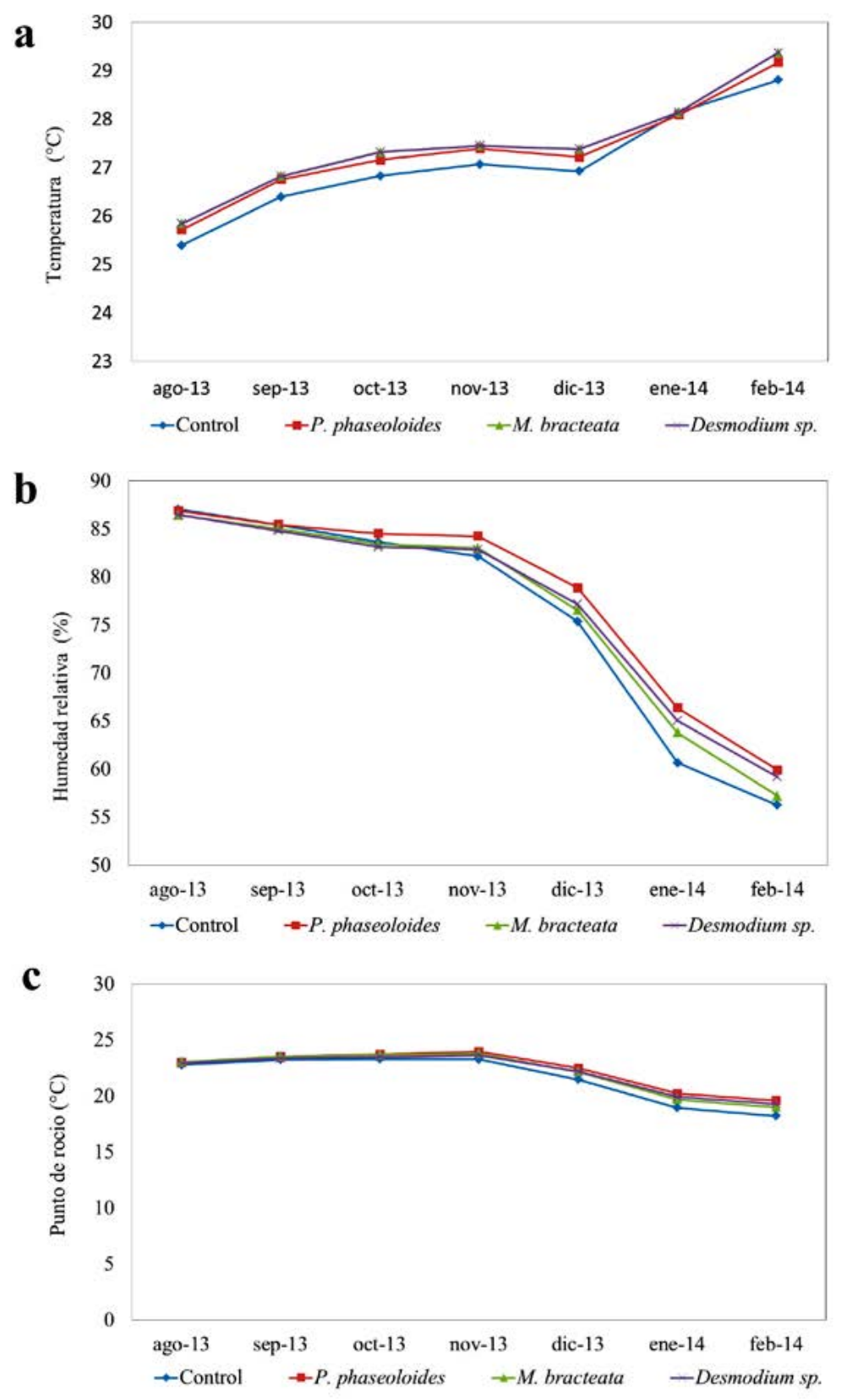

Figura 1. Variables climáticas evaluadas dentro de las parcelas experimentales a la altura de $1.20 \mathrm{~m}$ después de tres meses de establecidas las coberturas. A. Reporte de temperatura en cada uno de los tratamientos. B. Reporte de humedad relativa en cada uno de los tratamientos. C. Reporte de punto de rocío en cada uno de los tratamientos.

el tratamiento con $M$. bracteata el de mayor acumulación $(2.23 \pm 0.19 \mathrm{C} / \mathrm{N})$ con respecto al testigo, sin observar diferencias estadísticamente significativas, para lo cual se hace necesario un mayor número de muestras. El aumento del carbono orgánico fue bajo con respecto a los controles, el promedio de materia orgánica se conservó en los testigos $(1.94 \pm 0.2 \mathrm{C} / \mathrm{N})$. En tanto que en los tratamientos correspondientes a las tres coberturas el promedio de las réplicas estuvo levemente por encima para $M$. bracteata $(2.12 \pm 0.19 \mathrm{C} / \mathrm{N})$, P. phasoloides $(2.12 \pm 0.09 \mathrm{C} / \mathrm{N})$ y Desmodium $\mathrm{sp}$ 
$(2.236 \pm 0.19 \mathrm{C} / \mathrm{N})$. Los resultados obtenidos evidencian que en 10 meses en este tipo de suelo comienza a percibirse el aporte de materia orgánica por parte de las coberturas. La relación $\mathrm{C} / \mathrm{N}$ de los controles sin cobertura fue de 5.42 \pm 0.96 ; para $M$. bracteata de 5.80 \pm 0.35 ; para Desmodium sp. de $5.90 \pm 0.58$; y P. phaeoloides de 5.65 \pm 058 .

\section{Desarrollo de los árboles del clon RRIM-600 en la parcela experimental}

La evaluación del impacto del uso de coberturas sobre el desarrollo de los árboles realizado a través de comparación de la variable perímetro del tallo no mostró diferencias significativas respecto al control o a cualquiera de las tres coberturas (figura 2a). En cuanto que a la variable altura total sí se encontraron diferencias significativas entre las coberturas y el testigo $(p=0.029, F=3.008)$. Se observó que $M$. bracteata tuvo un efecto levemente mejor frente al testigo (figura 2b) (Dunnett $\mathrm{p}=0.009$ ), en comparación con Desmodium (Dunnett $\mathrm{p}=0.148$ ) y P. phaeoloides (Dunnett $p=0.336$ ), los cuales presentaron comportamiento similar.
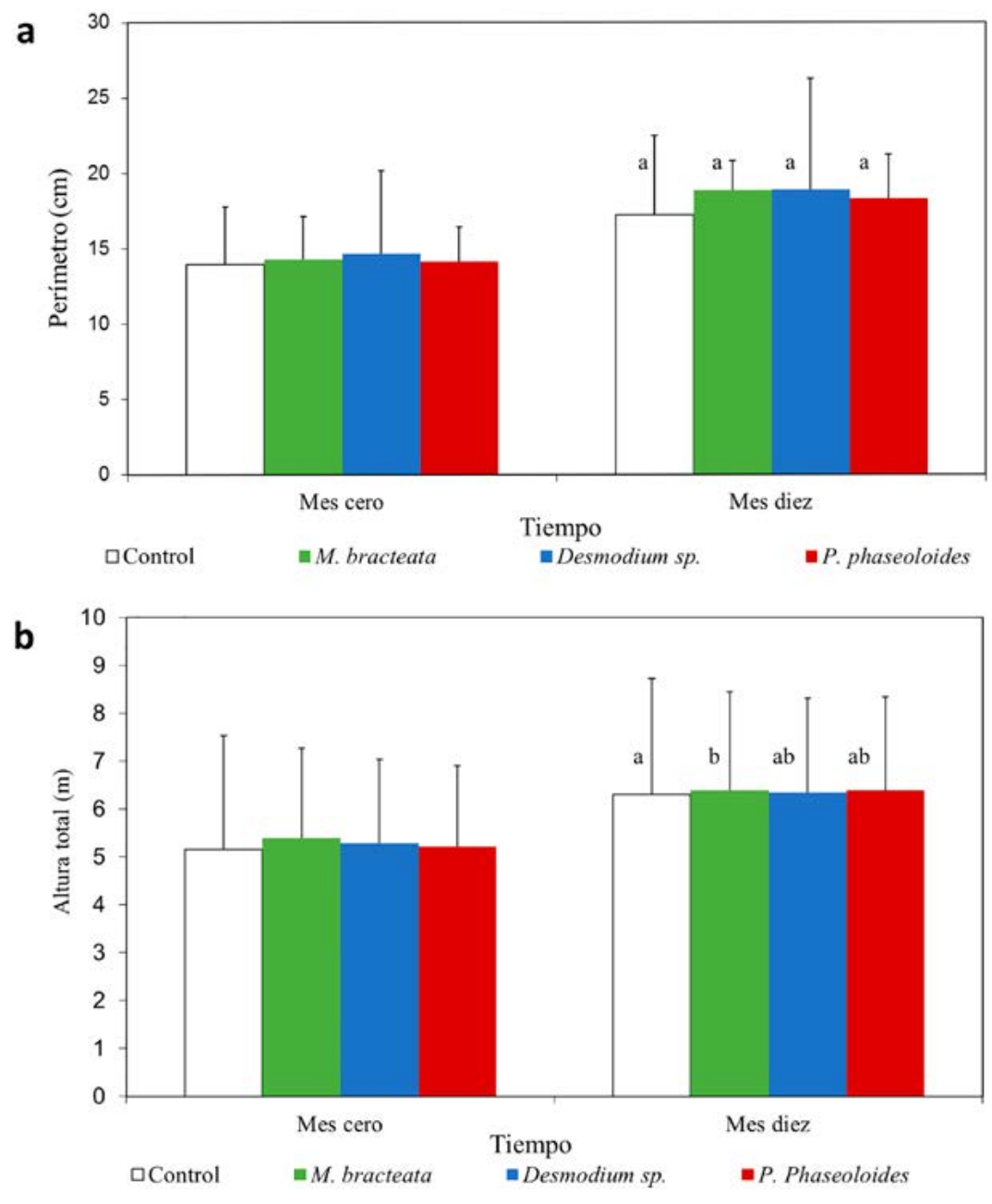

Figura 2. A. Perímetro de los árboles medidos al tiempo cero y 10 meses después de establecidas las coberturas respecto al control sin coberturas. B. Altura total de los árboles medidos al tiempo cero y 10 meses después de establecidas las coberturas respecto al control sin coberturas. Letras diferentes indican diferencias significativas. 


\section{Recuento de inóculos potenciales de hongos presentes en las coberturas en campo}

Los dos hongos que presentaron mayor cantidad UFC. $\mathrm{g}^{-1}$ de masa foliar en las tres coberturas durante el tiempo de evaluación correspondieron a Cladosporium sp. (Max: $1 \times 10^{5} \pm 3 \times 10^{4}$ en $P$. phaseolides, en febrero) y Epicoccum sorghi (Max: $5.8 \times 10^{4} \pm 1.4 \times 10^{4}$ en Desmodium sp., en noviembre) (figuras $3 a$ y $3 b)$.

El tercer hongo más abundante correspondió a Fusarium sp. (figura 3c). El cual se encontró en

\section{$\mathbf{a}$}

Cladosporium sp.

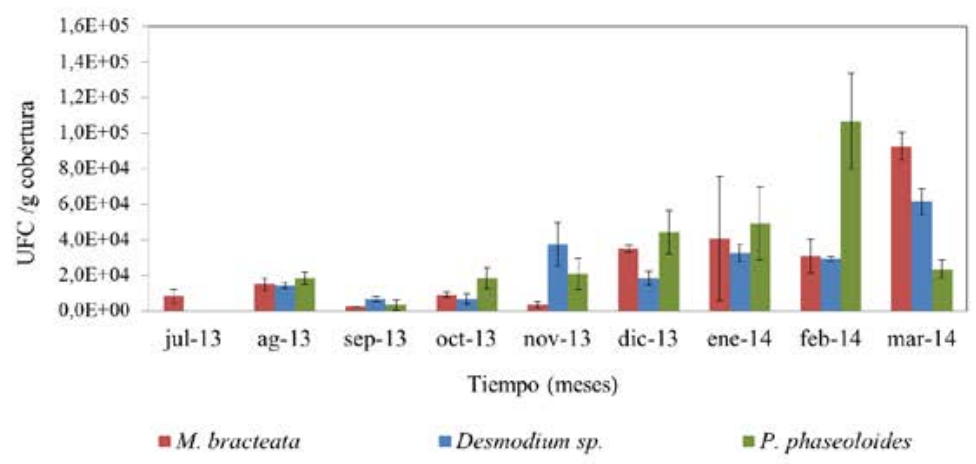

b

Epicoccum sorgui

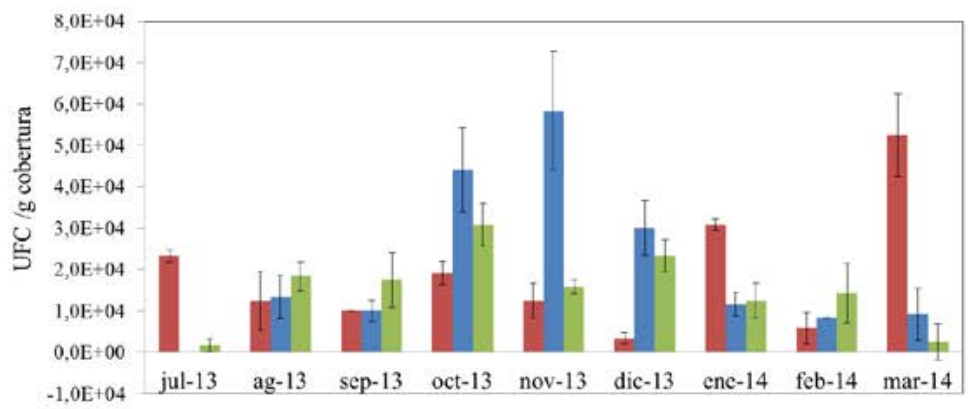

Tiempo (meses)

- M. bracteata

- Desmodium sp.

$=P$. phaseoloides

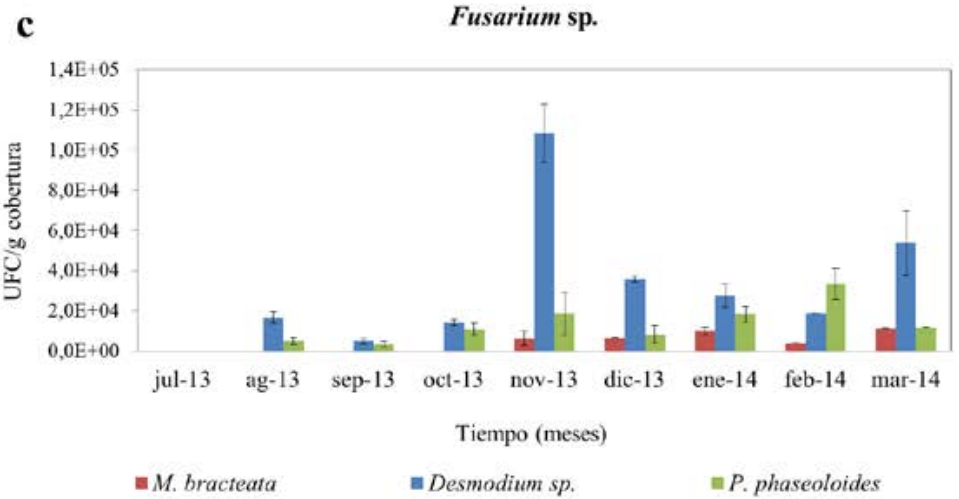

Figura 3. Principales hongos aislados e identificados en el follaje de las coberturas. A. UFC/g de cobertura de Cladosporium spp. B. UFC/g de cobertura de Epicoccum sorgui. C. UFC/g de cobertura de Fusarium sp. 
concentraciones más altas en Desmodium sp. (Max: $1.1 \times 10^{5} \pm 1.4 \times 10^{4}$, en noviembre) en la época hú-

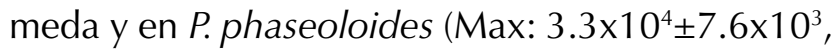
en febrero). Su presencia fue menor en $M$. bracteata (Max: $1.1 \times 10^{4} \pm 0.0$, en marzo).

Otros hongos encontrados que pueden llegar a ser agentes patogénicos en árboles de caucho correspondieron a Curvularia sp. y Phomopsis sp.; además de Cochliobolus sativus encontrado en Desmodium sp. (tabla 1).

En cuanto a Colletotrichum sp., durante el tiempo de muestro en campo sobre las tres coberturas solo se detectó en $P$. phaseoloides con una concentración de $2.5 \times 10^{3}$ UFC. $\mathrm{g}^{-1}$ de cobertura evaluada durante la época seca en el mes de diciembre con una temperatura promedio del mes de $27^{\circ} \mathrm{C}, \mathrm{HR}$ $78.9 \%$ y punto de rocío de $22.5^{\circ} \mathrm{C}$.

Los datos obtenidos de los hongos identificados en las coberturas se compararon con los datos de vigilancia fitosanitaria realizados en el área foliar de los árboles de RRIM 600 presentes en las parcelas experimentales; actividad que hace parte del manejo integrado de la plantación. No se encontraron coincidencias entre los momentos de alta incidencia de patógenos en el cultivo y el estado fitosanitario de las coberturas.

\section{Pruebas de inoculación de las coberturas en condiciones de casa malla}

El análisis de la secuencia ITS completa mostró que los tres aislamientos utilizados para las pruebas de infección en casa malla correspondieron a C. gloesporioides (datos no mostrados). Los ensayos de infección en condiciones de casa malla evidenciaron que las tres coberturas son susceptibles a los tres aislamientos de C. gloesporiodes.

Los síntomas observados correspondieron a manchas necróticas; lo que posteriormente se confirmó eran causadas por C. gloesporioides mediante aislamiento desde las hojas con la sintomatología, verificación microscópica y molecular (datos no mostrados). La incidencia de la enfermedad fue menor en $M$. bracteata y Desmodium sp. que en $P$. phaseoloides ( $\mathrm{p}=0.00, \mathrm{~F}=15$. 95; figura 5). No se encontraron diferencias significativas $(p=0.84, F=0.174)$ en cuanto a la incidencia de la enfermedad causada por cada uno de los aislamientos (figura 4), es decir, no hubo evidencia de diferencia en cuanto a la virulencia de los aislamientos.

Tabla 1. Géneros de hongos aislados e identificados en el follaje de las tres coberturas vegetales evaluadas

\begin{tabular}{ccc}
\hline Mucuna bracteata & Desmodium sp. & Pueraria phaseoloides \\
\hline Cladosporium sp. & Chaetomella sp. & Aspergillus sp. \\
Coniothyrium aleuritis & Cladosporium sp. & Cladosporium sp. \\
Curvularia sp. & Cochliobolus sativus & Colletotrichum gloesporioides \\
Epicoccum sorghi & Coniothyrium aleuritis & Coniothyrium aleuritis \\
Fusarium sp. & Curvularia sp. & Curvularia sp. \\
Myrothecium sp. & Epicoccum sorghi & Epicoccum sorghi \\
Mucor sp. & Fusarium sp. & Fusarium sp. \\
Nigrospora sp. & Myrothecium sp. & Myrothecium sp. \\
Penicillium $\mathrm{sp}$. & Mucor $\mathrm{sp}$. & Mucor \\
Phomopsis sp. & Nigrospora sp. & Nigrospora sp. \\
& Penicillium $\mathrm{sp}$. & Penicillium sp.
\end{tabular}




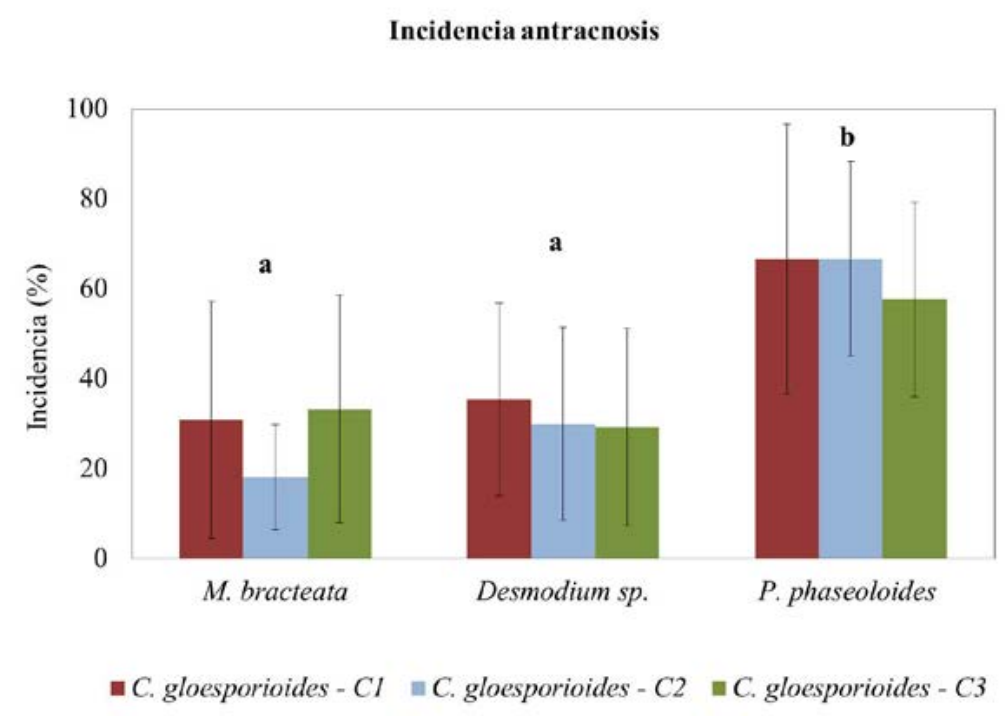

Figura 4. Incidencia de antracnosis en las tres coberturas evaluadas en condiciones de casa malla.
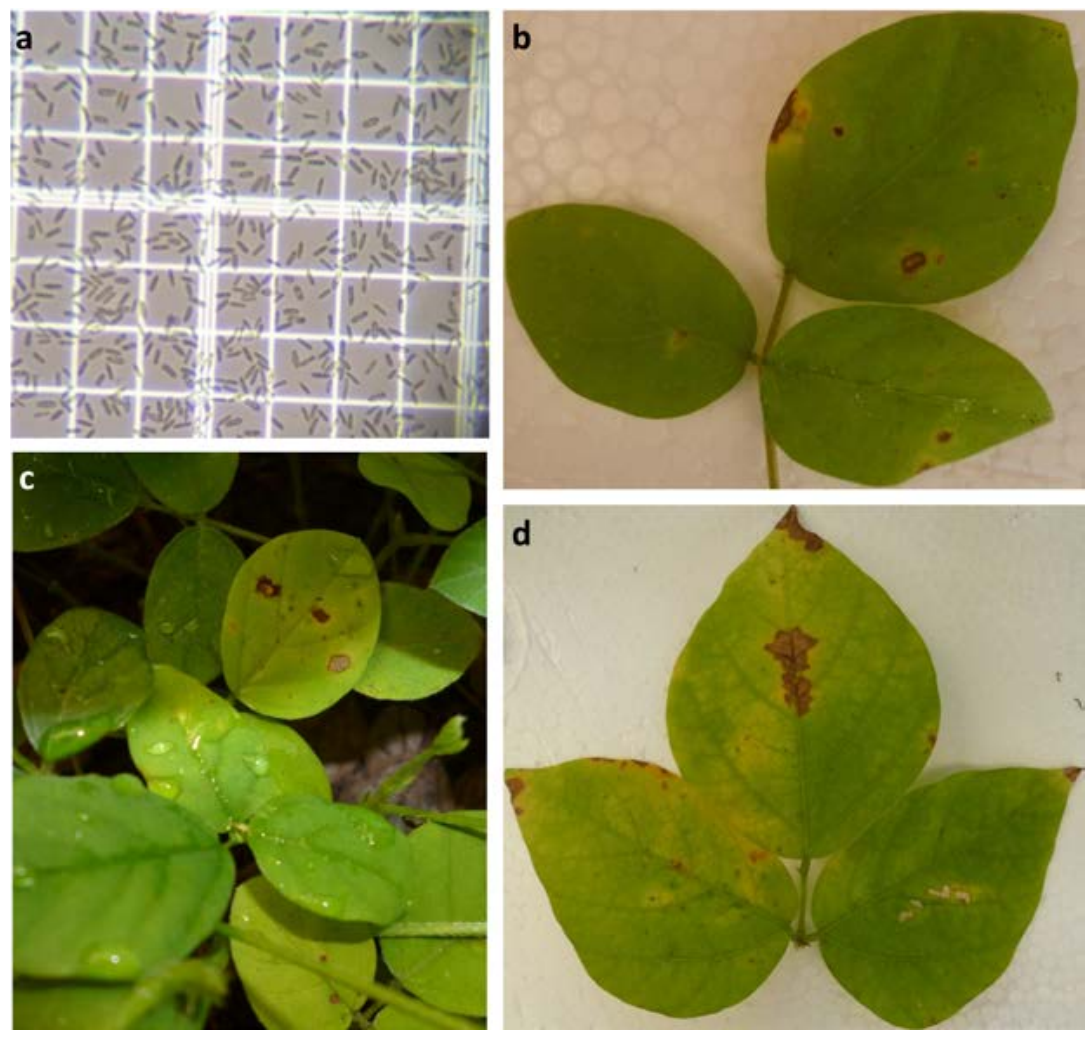

Figura 5. Inoculo y síntomas de antracnosis presentados por las coberturas en condiciones de casa malla. A. Conidias de C. gloesporioides obtenidas en cultivo in vitro usados para la inoculación de las tres coberturas, en condiciones de casa malla. B. M. bracteata con síntomas de antracnosis. C. Desmodium sp. con síntomas de antracnosis y D. P. phaseoloides con síntomas de antracnosis. 


\section{DISCUSIÓN}

En los suelos de la Altillanura el crecimiento de las Fabaceae aplicadas como coberturas presentan crecimiento competitivo con plantas arvenses establecidas en cultivos de Caucho y Palma. Como lo demuestran Pérez-Corpoica et al. (2002) con D. ovalifolium que en un periodo de seis meses puede llegar a cubrir un $72 \%$ de la superficie. En el caso del ensayo llevado a cabo en esta investigación, la cobertura que demoró más tiempo en establecerse fue Desmodium sp., la cual a los 10 meses de su siembra aun presentaba competencia con las arvenses. Pérez-Corpoica et al. (2002), también reportan que se pueden acortar estos tiempos disminuyendo la capacidad de germinación y multiplicación de las arvenses establecidas, mediante procesos de labranza y aplicación de herbicidas pre-emergentes y post-emergentes. Estas prácticas deben tener en consideración la no afectación de las plantas del cultivo establecido de caucho.

El tiempo estimado para el establecimiento de Mucuna en cultivos forestales de Malasia como palma de aceite es de 10 meses, en el cual la cobertura no permite el dominio de arvenses (Mathews, 1998); en el caso de $P$. phaseoloides el tiempo se ha estimado entre 9 y 13 meses con eliminación manual (Pinzón, Argel y Montenegro, 1985). Los resultados obtenidos son concordantes con los tiempos reportados por Pinzón et al. (1985), el tiempo tomado para su establecimiento total, entre el cultivo de caucho en el caso de $M$. bracteata fue de 9 meses y para $P$. phaseolides de 10 meses.

El análisis de los datos climáticos de temperatura y HR muestran que no hay diferencias significativas entre los tres microclimas creados por las tres coberturas estudiadas. Sin embargo, las medianas de los tratamientos muestran un leve aumento con respecto al control, lo que demuestra la generación de microclimas a la altura de 1.20 m, la cual es importante ya que a esta se traza el panel de rayado cuando los árboles entran en etapa de aprovechamiento. De acuerdo con la figura $1 \mathrm{~b}$, para HR se observa leve aumento (6\%) en las coberturas especialmente en $P$. phaseoloides con relación al control, lo cual se halló durante el periodo seco en los meses de noviembre a enero, contribuyendo a la protección de suelo en esta temporada (Villanueva y Guerra, 1987). En el caso de $M$. bracteata, reportan el aumento de la HR en el suelo de las parcelas que presentan la cobertura con relación a las que no la tienen. Este favorecimiento también podría promover la prevalencia de patógenos que limitan el cultivo de caucho como Colletotrichum sp., que además de afectar el follaje puede generar daños en el panel de rayado. Como se mencionó anteriormente, se ha reportado como el patógeno foliar más limitante para el cultivo del caucho en la Orinoquía (Castro, 2011) y, adicional a esto, capaz de infectar diferentes especies de los tres tipos de coberturas evaluados (Lenné, 1990; Mileo et al., 2006).

El porcentaje superior relativo de humedad retenida por $P$. phaseoloides en comparación a las otras dos coberturas evaluadas podría asociarse a las características de los foliolos y peciolos pubescentes. También, Clermont-Dauphin et al. (2018) demostraron que su establecimiento durante los primeros años de cultivos de caucho contribuye al aumento de la resistencia a sequía de los árboles. Sin embargo, es necesario tener en cuenta que esta cobertura no soporta periodos prolongados de estrés hídrico (Clermont-Dauphin, et al., 2016) y, por el contrario, algunas especies de pueraria en estas condiciones pueden generar incendios (Forseth e Innis, 2004).

El aporte realizado por las coberturas del tipo leguminosas al mejoramiento de la calidad del suelo se puede ver al analizar la relación $\mathrm{C} / \mathrm{N}$, una baja relación indica la rápida asimilación del nitrógeno y fijación por parte de la microflora del suelo (Broughton, 1977; Vrignon-Brenas et al., 2019). Los resultados obtenidos en este estudio antes y 10 meses después de establecidas las coberturas muestran una relación baja tanto en el tiempo cero $11.62 \pm 0.013$ como 10 meses después de la siembra que estuvo entre $5.42 \pm 0.96$ y $5.90 \pm 0.58$, tanto para las coberturas como para los controles. Se 
observó que la relación $\mathrm{C} / \mathrm{N}$ del tiempo cero declinó con respecto al mes 10, lo que puede deberse a un efecto de competencia de $H$. brasiliensis.

Investigaciones previas reportan relaciones $\mathrm{C} / \mathrm{N}$ por debajo de 20 para suelos de cultivos de caucho con leguminosas respecto a otras coberturas como gramíneas o coberturas naturales. Sin embargo, en este estudio no se observaron diferencias en las relaciones $\mathrm{C} / \mathrm{N}$ de suelos con las coberturas respecto a los controles sin coberturas, resultados similares fueron obtenidos por Barthès et al. (2004), quienes evaluaron el efecto del cultivo del maíz en asocio con Mucuna, el cultivo de maíz con fertilización tradicional y sin fertilización en la relación $\mathrm{C} / \mathrm{N}$ del suelo, encontrando que no hubo diferencias en los tratamientos al año de haber establecido el cultivo.

El efecto que tiene la incorporación de la biomasa de coberturas tipo leguminosa sobre la materia orgánica presente en el suelo ha sido ampliamente documentado para cultivos transitorios en países estacionarios (Fageria, Baligar y Bailey, 2005; Ding et al., 2006). En lo respectivo al aumento de la materia orgánica presente en el suelo, a partir de la asociación del cultivo con coberturas tipo leguminosas, también ha sido reportado, encontrándose un aumento mayor en los suelos de cultivos asociados con coberturas que en cultivos con fertilización tradicional o sin esta (Scholberg et al., 2010). En esta investigación se obtuvo un aumento del $0.29 \%$ en los suelos con coberturas Desmodium sp. y $M$. bracteata con relación al control; pero esta podría ir aumentado en años posteriores, como reporta Dinesh (2004), en cultivos de palma de coco evaluados durante 10 años en los cuales la materia orgánica se incrementa en factor de 2 a 3.

Yogartnam et al., (1977) encontraron que el establecimiento de leguminosas en combinación $P$. phaseoloides y Desmodium sp. aumentaron el diámetro de las plantas de caucho correspondientes al clon PB 59 desde los seis meses con respecto a las parcelas que no tenían coberturas. La evaluación del desarrollo de los árboles, Ilevada a cabo hasta el año cuatro, mostró diferencias de $13 \mathrm{~cm}$ aproximadamente entre los árboles establecidos con leguminosas con relación a las parcelas que no las tenían.

Dongsansuk et al. (2016) reportan un mayor crecimiento perimetral de árboles de caucho de dos años de edad asociados con $M$. bracteata con respecto al control sin cobertura y Clermont-Dauphin, et al. (2016) también encontraron diferencias significativas en el crecimiento perimetral y altura de los árboles de caucho asociados con $P$. phaseoloides con relación a los establecidos con otras coberturas y al control sin estas. Los árboles evaluados en nuestro experimento no mostraron diferencias significativas entre tratamientos, puede deberse a las coberturas se establecieron cuando los árboles ya tenían dos años de edad y al tiempo de evaluación.

Al comparar el diámetro de los árboles obtenidos en el estudio de Yogartnam et al. (1977), a los tres años de edad los árboles presentaron un perímetro aproximado de 16.07 cm y los árboles de nuestras parcelas control sin coberturas de 17 $\mathrm{cm}$, mostrando resultados similares del desarrollo de los árboles sin coberturas.

Se observaron diferencias significativas en cuanto a la altura total de los árboles, habiendo un efecto positivo de las coberturas con relación al control. Los resultados obtenidos nos permiten sugerir que en años siguientes será más evidente el impacto positivo que tienen las coberturas sobre el crecimiento de los árboles de caucho. En el caso de $H$. brasiliensis en la altillanura colombiana se hace necesario la evaluación de los mismos parámetros en periodos posteriores con el fin de definir el aporte al desarrollo de los árboles en un periodo de tiempo más amplio con las coberturas ya completamente establecidas.

Además de los beneficios ampliamente documentados del uso de las coberturas dentro de cultivos permanentes, en cuanto al mejoramiento de las propiedades físicas y químicas del suelo y el impacto positivo sobre el crecimiento de los árboles, también estimulan la biodiversidad de la biomasa microbiana del suelo y permiten algún grado 
de protección frente a patógenos siempre y cuando estas no sean hospederos alternos de patógenos limitantes del cultivo principal (Larkin, 2015).

En este estudio la identificación y cuantificación realizada de hongos presentes en el follaje de las tres coberturas a través de nueve muestreos en el tiempo mostró que los hongos más abundantes correspondieron a E. sorgui y Cladosporum spp, considerados como no limitantes de cultivo del caucho, a diferencia del tercer hongo encontrado correspondiente a Fusarium sp. el cual en los últimos años se ha asociado a necrosis del tallo, chancros y escurrimiento de látex (Huang et al., (2016). Estos resultados indican que el establecimiento de coberturas, especialmente Desmodium sp., en plantaciones de caucho en etapa productiva sería contraproducente ya que favorecería la disponibilidad de inoculo de Fusarium sp., el cual podría llegar a afectar el panel de sangría (Li et al., 2014). Se plantea esta hipótesis teniendo en cuenta que los dattalogers fueron ubicados a la altura de $1.20 \mathrm{~m}$, encontrándose diferencias en la HR de hasta seis grados con relación al control condiciones que favorecerían el desarrollo de enfermedades sobre este.

Los resultados obtenidos en esta investigación muestran que las tres coberturas son susceptibles a los tres aislamientos de C. gloesporioides, inoculados en condiciones de casa malla (figura 4). A pesar de que en campo durante los 10 meses de seguimiento a los hongos presentes en las Coberturas solo se detectó en $P$. phaseoloides en una ocasión, hecho que también contrasta con las enfermedades identificadas en el canopy de los árboles de caucho que correspondió principalmente a antracnosis, de acuerdo con la información suministrada por los monitores de campo. Se plantea que el inóculo presente en la parte aérea de los árboles de caucho no es suficiente para causar enfermedad en las coberturas; sin embargo, este podría incrementarse al entrar los árboles en etapa adulta cuando los procesos de defoliación naturales del árbol se den.

\section{CONCLUSIONES}

Los resultados obtenidos en este estudio permiten sugerir el uso de coberturas vegetales como $M$. bracteata, P. phaseoloides y Desmodium sp. durante la etapa improductiva de los cultivos de caucho natural en la altillanura colombiana, ya que favorece el crecimiento de los árboles, el manejo de arvenses y la protección del suelo. A pesar de que las tres coberturas en los ensayos de casa malla presentaron susceptibilidad a los tres aislamientos de C. gloesporioides evaluados, en campo no se encontraron elevadas concentraciones de UFC o focos de antracnosis, pero sí concentraciones altas de UFC de otros posibles patógenos de caucho como Fusarium sp., especialmente en el periodo de lluvias.

\section{AGRADECIMIENTOS}

Agradecemos la financiación por parte del Fondo de Fomento Cauchero a través del proyecto "Evaluación del desarrollo de cultivos de caucho natural en estado improductivo asociados con tres tipos de coberturas vegetales en la altillanura colombiana".

\section{REFERENCIAS}

Barthès, B., Azontonde, A., Blanchart, E., Girardin, C., Villenave, C., Lesaint, S. y Feller, C. (2004). Effect of a legume cover crop (Mucuna pruriens var. utilis) on soil carbon in an ultisol under maize cultivation in southern Benin. Soil Use and Management, 20(2), 231-239.

Bhaskar, D. y Dey, S. K. (2010). Control of Imperata cylindrica (L.) Beauv. in rubber plantations-a review. Natural Rubber Research, 23(1/2), 109-117.

Broughton, W. J. (1977). Effect of various covers on soli fertility under Hevea Brasiliensis Muell. Arg. and on growth of the tree. Agro-Ecosystems, 3, 147-70.

Castro, N. O. M. (2011). Caracterización de los patosistemas foliares de importancia económica en 
caucho (Hevea Brasiliensis Müll. Arg) en la Altillanura estructural plana del Meta (Colombia) (tesis de Maestría en Ciencias Agrarias). Universidad Nacional de Colombia, Bogotá, Colombia. 74 p.

Cenicaucho-Corpoica. (2013). Modelo productivo para el cultivo del árbol de caucho natural en la Orinoquía. Zonas de escape y no escape al Mal suramericano de las Hojas de caucho. Bogotá: Ministerio de Agricultura y Desarrollo Rural. 179 p.

Clermont-Dauphin, C., Dissataporn, C., Suvannang, N., Pongwichian, P., Maeght, J. L., Hammecker, C. y Jourdan, C. (2018). Intercrops improve the drought resistance of young rubber trees. Agronomy for Sustainable Development, 38(6), 56

Clermont-Dauphin, C., Suvannang, N., Pongwichian, P., Cheylan, V., Hammecker, C. y Harmand, J. M. (2016). Dinitrogen fixation by the legume cover crop Pueraria phaseoloides and transfer of fixed $\mathrm{N}$ to Hevea brasiliensis impact on tree growth and vulnerability to drought. Agriculture, Ecosystems \& Environment, 217, 79-88.

Dinesh, R. (2004). Long-term effects of leguminous cover crops on microbial indices and their relationships in soils of a coconut plantation of a humid tropical region. Journal of Plant Nutrition and Soil Science, 167(2), 189-195.

Dongsansuk, A., Ayutthaya, S. I. N., Kaewjumpa, N. y Polthanee, A. (2016). Photosynthetic efficiency of PSII and growth of young rubber tree (Hevea brasiliensis) planted with mucuna (Mucuna bracteata) cover crop. Asia-Pacific Journal of Science and Technology, 21(3), 12-27.

Ding, G., Liu, X., Herbert, S., Novak, J., Amarasiriwardena, D. y Xing, B. (2006). Effect of cover crop management on soil organic matter. Geoderma, 130(3-4), 229-239.

https://doi.org/10.1016/j.geoderma.2005.01.019

Fageria, N. K., Baligar, V. C. y Bailey, B. A. (2005). Role of cover crops in improving soil and row crop productivity. Communications in Soil Science and Plant Analysis, 36(19-20), 2733-2757.

Furtado, E. (2008). Doencas das folhas e do caule da seringueira. En A. Alvarenga y C. Santana do Carmo (eds.), Vicosa. Brasil: Seringueira EPAMIG. 893p.
Gunnell, P. S. y Gubler, W. D. (1992). Taxonomy and morphology of Colletotrichum species pathogenic to strawberry. Mycologia, 84(2), 157-165.

Cherr, C. M., Scholberg, J. M. S. y McSorley, R. (2006). Green manure approaches to crop production. Agronomy Journal, 98(2), 302-319.

Huang, G. X., Zhou, X. M., Liu, X. B., Cai, J. M. y Li, B. X. (2016). First report of rubber tree gummosis disease caused by Fusarium solani in China. Plant Disease, 100(8), 1788-1788.

https://doi.org/10.1094/PDIS-10-15-1147-PDN

IBM Corp (Released 2013). IBM SPSS Statistics for Windows, Version 22.0. Armonk, NY: IBM Corp.

Forseth, I. N. y Innis, A. F. (2004). Kudzu (Pueraria montana): history, physiology, and ecology combine to make a major ecosystem threat. Critical reviews in plant sciences, 23(5), 401-413.

Larkin, R. P. (2015). Soil health paradigms and implications for disease management. Annual Review of Phytopathology, 53, 199-221.

Lenné, J. M. y Stanton, J. M. (1990). Diseses of Desmodium Species-a Review. Tropical Grasslands, 24, $1-24$.

Leslie, J. F., Summerell, B. A. y Bullock, S. (2006). The Fusarium laboratory manual. Vol. 2, n. ${ }^{\circ} 10$. Ames, IA: Blackwell Pub.

Li, B. X., Shi, T., Liu, X. B., Lin, C. H. y Huang, G. X. (2014). First report of rubber tree stem rot caused by Fusarium oxysporum in China. Plant Disease, 98(7), 1008-1008.

Lubbe, C. M., Denman, S., Cannon, P. F., Groenewald, J. Z., Lamprecht, S. C. y Crous, P. W. (2004). Characterization of Colletotrichum species associated with diseases of Proteaceae. Mycologia, 96(6), 1268-1279.

Manamgoda, D. S., Cai, L., McKenzie, E. H., Crous, P. W., Madrid, H., Chukeatirote, E. y Hyde, K. D. (2012). A phylogenetic and taxonomic re-evaluation of the Bipolaris-Cochliobolus-Curvularia complex. Fungal Diversity, 56(1), 131-144.

Manamgoda, D. S., Rossman, A. Y., Castlebury, L. A., Chukeatirote, E. y Hyde, K. D. (2015). A taxonomic and phylogenetic re-appraisal of the genus 
Curvularia (Pleosporaceae): human and plant pathogens. Phytotaxa, 212(3),175-198.

\section{http://dx.doi.org/10.11646/phytotaxa.212.3.1}

Mathews, C. (1998). The Introduction and Establishment of a New Leguminous Cover Crop, Mucuna bracteata under Oil Palm in Malasia. The planter (Malaysia), 74(868), 359-68.

Mileo, L. J., Bentes, J. L. S., Silva, J. F. y Christoffoleti, P. J. (2006). Plantas de cobertura de solo Como hospedeiras alternativas de Colletotrichum guaranicola. Planta Daninha, 24(4), 677-83.

Pérez-Vera, O. A., Yáñez-Morales, M. D. J., Alvarado-Rosales, D., Cibrián-Tovar, D. y García-Díaz, S. E. (2005). Fungi associated to eucalyptus, Eucalyptus grandis Hill: Maid. Agrociencia (MonteciIlo), 39(3), 311-318.

Pérez-Corpoica, R., Rincón-Corpoica, A., Cipagauta-Corpoica, M., Schmidt-CIAT, A., Plazas-CIAT, C. y Lascano-CIAT, C. (2002). Maquenque (Desmodium heterocarpon (L.) DC. Subsp. ovalifolium (Prain.) Ohashi CIAT 13651) Leguminosa de usos múltiples en sistemas agropecuarios en Colombia. Villavicencio, Colombia: Corporación Colombiana de Investigación Agropecuaria; Cali, Colombia: Centro Internacional de Agricultura Tropical. 31 p.

Peberdy, J. F. (ed.) (2013). Penicillium and Acremonium (vol. 1). Springer Science \& Business Media.

Pinzón, R. B., Argel, J. P. y Montenegro, R. (1985). Control de malezas en el establecimiento de kudzú tropical. Pasturas tropicales, 7(2), 6-8.

Scholberg, J. M. S., Dogliotti, S., Leoni, C., Cherr, C. M., Zotarelli, L. y Rossing, W. A. H. (2010). Cover crops for sustainable agrosystems in the Americas. En E. Lichtfouse (ed.), Genetic engineering, biofertilisation, soil quality and organic farming (pp. 23-58). Sustainable agriculture reviews, vol 4. Netherlands: Springer.

Samarappuli, L., Karunadasa, P., Mitrasena, U. y Shantha, N. (2003). Mucuna bracteata: Ideal cover crop for efficient soil and water management in rubber cultivation. Tropical Agricultural Research and Extension, 6, 85-90.

Sanger, F. y Coulson, A. R. (1975). A rapid method for determining sequences in DNA by primed synthesis with DNA polymerase. Journal Molecular Biology, 94(3), 441-448.

Sreenivasaprasad, S. y Talhinhas, P. (2005). Genotypic and phenotypic diversity in Colletotrichum acutatum, a cosmopolitan pathogen causing anthracnose on a wide range of hosts. Molecular plant pathology, 6(4), 361-378.

Stirling, D. (2003). DNA Extraction from Fungi, Yeast, and Bacteria. En N. J. Clifton y D. Stirling (eds.), Methods in molecular biology PCR protocols (pp. 5354). Totowa, Nueva Jersey, EE. UU.: Humana press.

Villanueva, A. G. y Guerra, J. M. (1987). Cobertura Kudzú en plantaciones de palma. Siembra y DesarroIlo. Palmas, 8(4), 23-29.

Vrignon-Brenas, S., Gay, F., Ricard, S., Snoeck, D., Perron, T., Mareschal, L. y Malagoli, P. (2019). Nutrient management of immature rubber plantations. A review. Agronomy for sustainable development, 39(1), 11.

https://doi.org/10.1007/s13593-019-0554-6

Weir, B. S., Johnston, P. R. y Damm, U. (2012). The CoIletotrichum gloeosporioides Species Complex. Studies in Mycology, 73, 115-80.

Walkley, A. y I. A. Black. (1934). An examination of the Degtjareff method for determining soil organic matter, and proposed modification of the chromic acid titration method. Soil Science, 37, 29-38.

White, T. J., Bruns, T., Lee, S. J. W. T. y Taylor, J. (1990). Amplification and direct sequencing of fungal ribosomal RNA genes for phylogenetics. En M. Innis, D. H. Gelfand, J. J. Sninsky y T. J. White (eds.), PCR protocols: guide to methods and applications (pp. 315-322). San Diego, California: Academic Press

Yogartnam, N., Sulaim, H., Karunaratna, A. D. M. y Peiris, K. S. A. (1977). Management of covers under Hevea in Sri Lanka. Journal Rubber Research Institute Sri Lanka, 54, 291-298.

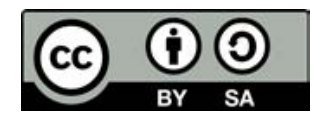

